\title{
PCAtest: Testing the statistical significance of Principal Component Analysis in $\mathbf{R}$
}

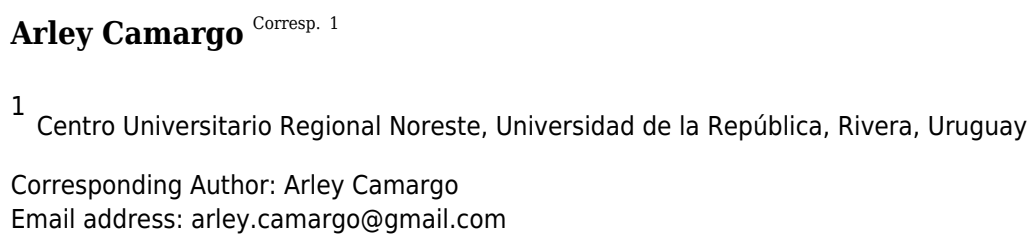

Principal Component Analysis (PCA) is one of the most broadly used statistical methods for the ordination and dimensionality-reduction of multivariate datasets across many scientific disciplines. Trivial PCs can be estimated from data sets without any correlational structure among the original variables, and traditional criteria for selecting non-trivial PC axes are difficult to implement, partially subjective or based on ad hoc thresholds. PCAtest is an $\mathrm{R}$ package that implements permutation-based statistical tests to evaluate the overall significance of a PCA, the significance of each PC axis, and of contributions of each observed variable to the significant axes. Based on simulation and empirical results, I encourage R users to routinely apply PCAtest to test the significance of their PCA before proceeding with the direct interpretation of PC axes and/or the utilization of PC scores in subsequent evolutionary and ecological analyses. 
1 PCAtest: Testing the statistical significance of Principal Component Analysis in R

2

3 Arley Camargo

4

5 Centro Universitario Regional Noreste, Sede Rivera, Universidad de la República, Ituzaingó 667,

6 Rivera 40000, Uruguay. E-mail: arley.camargo@gmail.com

7

8 Running headline: Statistical significance of PCA 
9 Abstract

10

11 Principal Component Analysis (PCA) is one of the most broadly used statistical methods for the

12 ordination and dimensionality-reduction of multivariate datasets across many scientific

13 disciplines. Trivial PCs can be estimated from data sets without any correlational structure

14 among the original variables, and traditional criteria for selecting non-trivial PC axes are difficult

15 to implement, partially subjective or based on ad hoc thresholds. PCAtest is an R package that

16 implements permutation-based statistical tests to evaluate the overall significance of a PCA, the

17 significance of each PC axis, and of contributions of each observed variable to the significant

18 axes. Based on simulation and empirical results, I encourage R users to routinely apply PCAtest

19 to test the significance of their PCA before proceeding with the direct interpretation of PC axes

20 and/or the utilization of PC scores in subsequent evolutionary and ecological analyses.

21

22 Keywords

23

24 Principal Component Analysis, statistical significance, permutation, R package, PCAtest 


\section{Introduction}

26

27 Principal Component Analysis (PCA), originally developed by Pearson (1901) and Hotelling

28 (1933), is one of the most broadly used statistical methods for the ordination and dimensionality-

29 reduction of multivariate datasets across many scientific disciplines with many adaptations for

30 different goals and data types (Manly, 1986; Jackson, 1991; Vieira, 2012; Jolliffe \& Cadima,

31 2016). PCA estimates independent, linear combinations of the original (possibly correlated)

32 variables in such a manner that the first component (PC1) accounts for the largest possible

33 variance in the data, and each subsequent PC accounts for decreasing proportions. In most

34 applications, PC scores (also called z-scores or 1-scores; see Jackson, 1991) are calculated for the

35 observations based on those PC axes considered to adequately account for the correlational

36 structure in the data. These PC scores are used to plot and find groupings of the observations in

37 bi- or tri-dimensional spaces defined by the orthogonal PC axes (Manly, 1986; Jackson, 1991)

38 and also to look for potential associations with explanatory variables (e.g., correlation analysis

39 between PC scores obtained from morphological variables and environmental variables of the

40 same observations). In addition, the loading matrix is usually inspected to find out which of the

41 original variables contributed the most to each of the relevant PCs (Manly, 1986; Jackson, 1991;

42 Vieira, 2012).

43

44 It is well known that random (or trivial) PCs can be estimated from data sets without any

45 correlational structure among the original variables due to sampling error, especially when

46 number of observations is low relative to the number of variables (Manly, 1986; Jackson, 1993;

47 Vieira, 2012). Even when there is correlation among the original variables, it is necessary to 
48 retain the PC axes that summarize that real structure for downstream inferences, and discard the

49 PCs that do not account for more variation than that expected by chance due to sampling error,

50 which creates spurious correlation among the original variables. The criteria traditionally applied

51 for selecting the number of PC axes to be retained in subsequent analyses are difficult to

52 implement, partially subjective or based on ad hoc thresholds (Vieira, 2012). For instance, a

53 traditional 'rule-of-thumb' criterion considers that PC axes with eigenvalues larger than one

54 should be retained because they explain more variation than that of a single original variable.

55 Another criterion found in the literature suggests that one should retain as many PC axes as

56 necessary to account for most of the variation in the data, which usually leads to the retention of

57 random axes that explain small portions of variance. Finally, a popular approach consists in

58 visually inspecting a scree plot to find an "elbow" in the distribution of observed eigenvalues

59 that represents the point at which eigenvalues become small and level off as expected from

60 random noise. In general, when following these criteria, it becomes very difficult to ascertain if a

61 given PC axis should be retained when its eigenvalue is very close to that one expected by

62 random chance (Vieira, 2012). Because these ad hoc procedures are strongly dependent on

63 distributional assumptions, permutation-based statistical tests are ideal for evaluating the

64 significance of PCA (Manly, 1986; Jackson, 1993; Vieira, 2012). Empirical evidence and

65 simulation studies have shown the permutation-based tests (or more generally, 'parallel

66 analysis') are the most consistently accurate under a range of conditions (Manly, 1986; ter Braak,

67 1988; Dijksterhuis \& Heiser, 1995; Peres-Neto, Jackson \& Somers, 2005). Therefore,

68 permutational methods are ideal when one needs to select those PCs representing systematic

69 sources of variation in multivariate data and discard other PCs that only reflect random noise

70 caused by sampling error. 
71

72 A permutation-based test consists in shuffling individual values within each observed variable to

73 break the original correlation structure among variables without affecting their distributions,

74 calculating the test statistic for each permutation to build a null distribution, and to compare this

75 distribution with the observed statistics (ter Braak, 1988; Dijksterhuis \& Heiser, 1995; Perez-

76 Neto et al., 2005; Vieira, 2012; Vitale et al. 2017). Consequently, the null hypothesis that the

77 variables used in the PCA are uncorrelated with each other can be tested with null distributions

78 of test statistics generated via data permutation. Vieira (2012) proposed to evaluate the

79 significance of PCA with two test statistics that summarizes variation in eigenvalues alone $(\psi)$ or

80 in combination with the number of variables ( $\varphi$; Gleason \& Staelin, 1975). If these statistics

81 show that a PCA is meaningful, then null distributions can also be used to select the number of

82 significant PC axes using for instance the rank-of-roots statistic (ter Braak, 1988). Finally,

83 Björklund (2019) also suggested to use Vieira (2012)'s index of the loadings to test the

84 significance of the contributions of the original variables (eigenvector loadings) to each

85 significant PC also via permutation. Vieira (2012) implemented these tests of PCA significance

86 in the proprietary commercial Matlab program (typically used by mathematicians and engineers),

87 but an equivalent application in open-source free R software (R Core Team, 2021), more broadly

88 used in life and environmental sciences, is still lacking. While there are $\mathrm{R}$ packages

89 implementing 'parallel analysis' to select the number of PCs, such as $n$ Factors (Raîche \& Magis, 90 2020), there are no implementations for testing the overall significance of a PCA or the loadings

91 of each significant PC.

92

93 Material \& Methods

Peer] reviewing PDF | (2021:08:64827:3:0:NEW 28 Jan 2022) 
94

95 Herein, I introduce an R package to perform the statistical tests proposed by Vieira (2012) to

96

97 98

100

101

102

103

104

105

106

107

108

109

110

111

112

113

114

115

116

evaluate the overall significance of a PCA, the significance of each PC axis, and of the variable

loadings for the significant axes. This R package is named PCAtest and is available for download from github.com/arleyc/PCAtest. Eigenvalues and eigenvector loadings are calculated from a standardized (centered and scaled) multivariate matrix (variables in columns and observations in rows) with the stats:prcomp function, which performs a singular value decomposition of the correlation matrix (Choi \& Yang, in press). Observed eigenvalues are used to calculate empirical $\psi$ and $\varphi$ values, percentages of explained variance for each PC axis (rank-of-roots statistic; ter Braak, 1988), and the indexes of the loadings of each variable on each PC axis (Vieira, 2012). Next, PCAtest performs a bootstrap resampling of the observed data using base::sample to sample rows from the data set with replacement and calculates all statistics for each bootstrap replicate. Bootstrap resampling is a classical and well-established numerical methodology for calculating confidence intervals of statistics based on approximation of their sampling distributions (Efron, 1979; Efron \& Tibshirani, 1986; Manly, 1991). To build null distributions for each statistic, PCAtest permutes observations within each variable by sampling without replacement within columns (i.e., applying base::sample to all columns with base::apply), and calculates all statistics for each random permutation. By default, the function runs 1,000 random permutations and bootstrap replicates of the empirical data. Based on the bootstrap resampling and permutation, 95\%-confidence intervals around mean values are calculated with stats::quantile. Finally, $P$-values are calculated as the fraction of null statistics larger than the observed statistics in relation to the number of random permutations. Significant $P$-values for $\psi$ and $\varphi$ imply that there is non-random correlational structure in the data, and that a PCA is 
117 biologically meaningful. Significant eigenvalues mean that the respective PC axes reflect non-

118 random correlations among variables, and significant loadings mean that the respective variables

119 have a larger contribution in the PC score beyond random noise. The nominal alpha level by

120 default is set to 0.05 , but it can be changed by the user to explore the effects of more

121 conservative $v s$. liberal Type I error.

122

123 The function creates a list object including the following elements: (1) the empirical $\psi$ and $\varphi$

124 values, (2) the $\psi$ and $\varphi$ values derived from permutation, (3) the percentage of variation

125 explained by each empirical PC, (4) the percentage of variation explained by each PC with the

126 permuted and bootstrapped data, (5) the index loadings of each PC with the permuted and

127 bootstrapped data, and optionally, (6) the correlations of each PC with the original variables

128 using the empirical, the permuted, and the bootstrapped data. Messages are displayed in the

129 console during the run informing the user about the progress of the analysis at each major step:

130 bootstrap replication, random permutation, and statistical tests. After completing these steps, the

131 function displays a summary of the data set dimensions, the randomization results, and the

132 recommendations for subsequent analyses. First, the output describes the size of the dataset and

133 the number of permutations and bootstraps. Next, the randomization results for each statistic and

134 eigenvalue are displayed including the empirical value, the range of randomized values, and the

135 corresponding $P$-value. Based on these results, the output informs how many PC axes are

136 significant and how much percentage of the total variation they account for (mean and 95\%-

137 confidence interval). Finally, based on the randomization of PC loadings, the output lists the

138 variables with significant loadings for each of the retained PC axes. The user can also choose to

139 calculate the correlations of each PC axis with each of the observed variables as an alternative to 
140 the index of the loadings, which are prone to high Type I error in large data sets (Vieira, 2012). If

141 the empirical $\psi$ and $\varphi$ statistics are not significant, the function reports the results of these

142 randomization tests only.

143

144 The function produces four types of plots: (1) the null distributions of randomized $\psi$ values and

145 the empirical value, (2) the null distribution of randomized $\varphi$ values and the empirical value, (3)

146 the percentage of total variation explained by each randomized and empirical PC (mean and

$14795 \%$-confidence intervals), and (4) the index loadings of the randomized and empirical values of

148 significant PC axes (mean and 95\%-confidence intervals). The function creates up to four plots

149 in a single page, with additional plots shown in as many new pages as necessary given the

150 number of significant PCs. Empirical statistics (means and confidence intervals based on

151 bootstrap resampling) are plotted in red color and null statistics based on random permutation

152 (means and confidence intervals) are plotted in gray color. The user can opt to suppress the plots

153 that are produced by default.

154

\section{Results}

156

157 To demonstrate the use of the PCAtest, I simulated several datasets with varying levels of

158 correlation structure among the variables and reanalyzed more complex datasets found in the

159 literature. The implementation of these analyses is included in the package and it is also available 160 as a tutorial in the website arleyc.github.io/PCAtest/. 
162 I simulated three datasets consisting of five variables with 100 observations and varying levels

163 of correlation among the variables $(\mathrm{r}=0,0.25$, and 0.5$)$. These artificial datasets ( 100 replicates

164 for each value of $\mathrm{r}$ ) were generated with the R function MASS:mvrnorm specifying a mean equal

165 to zero $(\mathrm{mu}=0)$ and the covariance matrix (Sigma) with variances $=1$ and covariances $=r$ for all

166 five normally-distributed variables. In the first case, a PCA should not find any significant

167 correlation structure among the variables. In the second and third examples, the PCA should be

168 significant and PC1 should capture most of the variance because the simulated correlation

169 structure is identical among all variables. In the first example, PCAtest did not detect any

170 significant correlation structure as expected in $95 \%$ of the replicates (Fig. 1, Table S1). In the

171 second example, the first PC axis was significant in $100 \%$ of the replicates, while the second PC

172 axis was also significant in one replicate (Fig. 2, Table S2). In the third example, the first PC axis

173 was significant in $100 \%$ of the replicates (Fig. 3, Table S3).

174

175 I also re-analyzed a published dataset with PCAtest to emphasize the need for testing non-

176 randomness of PCA axes by assessing sampling variance in the empirical data. Recently, Wong

177 \& Carmona (2021) used a PCA analysis to reduce dimensionality of a dataset consisting of seven

178 morphological variables of 29 ant species. They retained the first two PC axes based on the

179 selection criterion that eigenvalues greater than one are biologically-meaningful, and then used

180 the PC scores from these axes in ecological analyses of functional diversity. The re-analysis

181 shows that the first two eigenvalues are greater than one (3.85 and 1.52 for PC1 and PC2,

182 respectively), and the significant $\psi$ and $\varphi$ statistics suggest that a PCA is able to extract non-

183 random correlation structure among the morphological variables (Fig. 4A, B; Table S4).

184 However, the randomization test with the 'rank-of-roots' statistic (percentage of explained 
185 variance $)$ indicates that PC2 is not significant $(P$-value $=0.16$, Fig. $4 C)$. Furthermore, the

186 variable 'eye width' does not contribute a significant loading to PC1 (Fig. 4D, variable 6).

187 Consequently, Wong and Carmona (2021) should only have used the PC1 and ignored the

188 contribution of the 'eye width' variable to the scores of this PC axis in their ecological analyses.

189 While the inclusion of non-significant PC axes and variables might not have impacted the

190 outcome of their analyses, it probably added additional noise and uncertainty to the interpretation

191 of their results.

192

193 In order to exemplify a data set with more variables than observations, I also re-analyzed the

194 microarray data of Ringnér (2008) containing the expression profiles of 8,534 genes screened in

195105 samples (Data S1). The author did not evaluate the significance of the PCA, but used the

196 first two PCs to project the samples in a bi-dimensional scatter plot, which together accounted

197 for only $18.7 \%$ of the total variation. The analysis with PCAtest using 1,000 random

198 permutations found significant both $\psi$ and $\varphi$ values (Fig. 5A, B) and 21 significant PC axes

199 accounting for $63.4 \%$ of the total variation (Fig. 5C). While the index of the loadings found

2007,419 variables (i.e., genes) contributing significantly to PC1, only a subset of 2,612 variables

201 had significant correlations with this PC. These results suggest that the index of the loadings

202 falsely finds too many significant results for irrelevant variables in large data sets as Vieira

203 (2012) found with simulated data. Given the complexity of this dataset with many more variables

204 than observations, I transposed the matrix using the function base::t to run a Q-mode PCA

205 analysis sensu Lee et al. (2017), which resulted in six significant PCs accounting for 79\% of the

206 total variance (Fig. 6, Table S5). Moreover, PC1 alone explained 67\% suggesting a strong

207 correlation structure, meaning a shared gene expression profile among samples, and that PC1 
208 scores from the Q-mode analysis could be more informative for discovering gene groupings than

209 the R-mode PCA used by Ringnér (2008).

210

\section{Discussion}

212

213 The overall significance of a PCA analysis has been poorly addressed in mainstream

214 evolutionary biology journals (Björklund, 2019). However, the lack of a rigorous statistical

215 evaluation of PCA significance is common place across a variety of scientific disciplines as

216 demonstrated in a systematic search of papers published during 2021 (until Nov $30^{\text {th }}$ ) in PeerJ

217 journals. Using the keyword 'PCA', I found that none of 155 articles evaluated the overall

218 significance of the PCA, and that only 26 (16.8\%) used an explicit criterion for PC selection

219 (Table 1, Table S6). Three criteria were applied to select the number of relevant PC axes, namely

220 the "cumulative variance", "eigenvalue $>1$ ", and "scree plot" criteria, although they are known

221 to suffer of drawbacks or are difficult to apply in practice. Often, these criteria lead to the

222 consideration of PC axes that only represent random noise in the data, but not correlation

223 structure among the original variables. This is the case with the criterion of including as many

224 PC axes as necessary to reach a pre-specified level of explained variation (Peres-Neto, Jackson

$225 \&$ Somers, 2005), which in most cases results in the consideration of trivial, non-significant axes.

226 In the literature review, I found that when this criterion was used $(14$ out of $155=9.0 \%)$, the cut-

227 off amount of cumulative variance was very different across articles (63-98\%, Table 1).

228 Similarly, the "greater-than-one" eigenvalue criterion, which was used in 11 articles (7.1\%),

229 sometimes results in the inclusion of non-significant axes, especially for data sets with many 
230 variables (Vieira, 2012). Similarly, the scree plot criterion, which tends to perform poorly with

231 an increasing number of variables (Vieira, 2012), was used in one article only $(0.7 \%$, Table 1$)$.

232

233 The performance evaluations of Vieira (2012) indicated that the chosen statistics used in PCAtest

234 had a good overall performance even in the case of "noisy" data sets containing variables with

235 low correlation or representing linear combinations of the other variables. In particular, the $\psi$

236 and $\varphi$ statistics performed very well even in the case of data sets with more variables than

237 observations. The preliminary evaluation of PCAtest with simulated datasets suggests a high

238 accuracy in detecting true correlational structure in the data, but a 5\% error when rejecting the

239 null hypothesis of no correlation structure (Type I error). Further exploration of parameter space

240 is necessary to evaluate the conditions (number of variables and observations, correlation

241 patterns among variables) under which PCAtest finds a significant PCA when there is no

242 correlational structure in the data. For the selection of PC axes, PCAtest implements the rank-of-

243 roots statistic, which always had better performance than alternative statistics (Vieira, 2012).

244 However, for the selection of relevant variables, the index of the loadings had a high false

245 positive rate in large data sets (i.e., selecting too many contributing variables with non-

246 significant loadings). Therefore, an option was included in PCAtest to calculate the correlations

247 of the PCs with the variables, in addition to the index of the loadings, for comparison of both

248 statistics. In addition, the problem of 'axis reflection' (i.e., the arbitrary permutation of signs

249 among loadings and PC scores), which is well known in the literature (Jackson, 1995; Mehlman,

250 Shepherd \& Kelt, 1995; Peres-Neto, Jackson \& Somers, 2003, 2005), is effectively avoided with

251 these two statistics as originally implemented by Vieira (2012) and in the R package PCAtest

252 introduced here. 
254 Traditional parametric, statistical approaches have been used to evaluate significance of a PCA, 255 which assume multivariate normality distribution of the data (Peres-Neto, Jackson \& Somers, 256 2005). Alternatively, non-parametric approaches such as the bootstrap have been used to 257 compare the confidence intervals of PCA statistics with their expected values based on ad criteria 258 as explained above (Peres-Neto, Jackson \& Somers, 2003). However, bootstrap techniques do 259 not build an appropriate null hypothesis because the resampling does not remove the correlation 260 structure among the original variables and do not account for axis reflection and reordering 261 (Vieira, 2012). In addition, cross-validation computational approaches have been frequently used

262 for PC selection, but they are considered to be more appropriate when the goal of the PCA is 263 data prediction instead of data exploration (Vitale et al., 2017). On the contrary, permutation 264 approaches that shuffle observations within variables, as implemented in PCAtest, effectively 265 creates an expected distribution of several statistics under the assumption of non-correlated 266 variables which can be used to evaluate the significance of a PCA, to retain the number of 267 relevant PC axes, and the identify the significantly contributing variables to those axes. Vitale et 268 al. (2017) introduced a novel permutational approach that uses the residual matrix for extracting 269 PCs (except for PC1), which increases the sensitivity in detecting relevant PC accounting for 270 small portions of variance. However, they did not provide a software implementation for this 271 alternative algorithm and the statistic they used (an F-like ratio-of-roots) showed high Type I 272 error for trivial PCs in simulations (Vieira, 2012). More recently, Dobriban (2020) rigorously 273 formalized the theoretical foundation of permutation-based, parallel analysis for selecting the 274 number of relevant PCs, and warned about the 'shadowing' effect of major PCs with large 275 eigenvalues that could obscure the signal of subsequent, weaker PCs. However, parallel analysis 
276 works well, especially in high-dimensional data sets, when a PCA is able to extract PCs with

277 loadings from several observed variables (Dobriban, 2020).

278

279 Conclusions

280

281 PCA is one of the most popular statistical approaches across many disciplines for data ordination 282 and dimensionality reduction of multivariate data. However, PCA is meaningful and should only 283 be applied when there is a significant correlation structure among the observed variables. Due to 284 sampling variance alone, it is possible that some level of random correlation structure could 285 occur in the data. Although it is necessary to evaluate if the observed correlation structure is 286 higher than expected due to chance alone, this evaluation is rarely undertaken in empirical 287 studies. The R package PCAtest uses random permutation to assess PCA significance and to 288 select the number of significant PCs. In addition, PCAtest can also test the contribution of the 289 observed variables to each significant PC. Therefore, I encourage R users to routinely apply 290 PCAtest to test the significance of their PCA analyses before proceeding with the direct 291 interpretation of PC axes and/or the utilization of PC scores in subsequent evolutionary and 292 ecological analyses.

293

\section{Acknowledgements}

295

296 I thank the comments and suggestions of two anonymous reviewers that greatly improved the

297 manuscript and the guidance of the Academic Editor for the preparation of the R package. 


\section{References}

300

301 Abdi H, Williams LJ. 2010. Principal component analysis. WIREs Computational Statistics

302 2:433-459. DOI: 10.1002/wics.101

303 Björklund M. 2019. Be careful with your principal components. Evolution 73:2151-2158. DOI:

304 10.1111/evo.13835

305 Bro R, Smilde AK. 2014. Principal component analysis. Analytical Methods 6:281-2831. DOI:

306 10.1039/C3AY41907J

307 Choi H, Yang X. In press. Asymptotic properties of correlation-based principal component 308 analysis. Journal of Econometrics. DOI: 10.1016/j.jeconom.2021.08.003.

309 Dijksterhuis GB, Heiser WJ. 1995. The role of permutation tests in exploratory multivariate data 310 analysis. Food Quality and Preference 6:263-270. DOI: 10.1016/0950-3293(95)00025-9

311 Dobriban E. 2020. Permutation methods for factor analysis and PCA. The Annals of Statistics 312 48:2824-2847. DOI: 10.1214/19-AOS1907

313 Efron B. 1979. Bootstrap methods: Another look at the Jacknife. The Annals of Statistics 7:1-26.

314 DOI: $10.1214 / \mathrm{aos} / 1176344552$

315 Efron B, Tibshirani R. 1986. Bootstrap methods for standard errors, confidence intervals, and 316 other measures of statistical accuracy. Statistical Science 1:54-75. DOI: $10.1214 /$ ss/1177013815

317 Gleason TC, Staelin R. 1975. A proposal for handling missing data. Psychometrika 40:229-252.

318 DOI: 10.1007/bf02291569

319 Jackson DA. 1993. Stopping rules in principal components analysis: A comparison of heuristical 320 and statistical approaches. Ecology 74:2204-2214. DOI: $10.2307 / 1939574$

321 Jackson JE. 1991. A User's Guide to Principal Components. New York: John Wiley \& Sons. 
322 Jolliffe IT, Cadima J. 2016. Principal Components Analysis: a review and recent developments.

323 Philosophical Transactions of the Royal Society A 374:20150202. DOI: 10.1098/rsta.2015.0202

324 Hotelling H. 1933. Analysis of a complex of statistical variables into principal components.

325 Journal of Educational Psychology 24:417-441. DOI: 10.1037/h0071325

326 Lee LC, Liong C-Y, Jemain AA. 2017. Q-mode versus R-mode principal component analysis for

327 linear discriminant analysis (LDA). AIP Conference Proceedings 1842, 030024. DOI:

$32810.1063 / 1.4982862$

329 Manly BJF. 1986. Multivariate methods. London: Chapman \& Hall.

330 Manly BJF. 1991. Randomization and monte carlo methods in biology. London: Chapman \& 331 Hall.

332 Mehlman DW, Shepherd UL, Kelt DA. 1995. Bootstrapping principal components analysis: A 333 comment. Ecology 76:640-643. DOI: 10.2307/1941219

334 Pearson K. 1901. On lines and planes of closest fit to systems of points in space. Philosophical 335 Magazine 2:559-572. DOI: 10.1080/14786440109462720

336 Peres-Neto PR, Jackson DA, Somers KM. 2003. Giving meaningful interpretation to ordination 337 axes: assessing loading significance in principal component analysis. Ecology 84:2347-2363.

338 DOI: $10.1890 / 00-0634$

339 Peres-Neto PR, Jackson DA, Somers KM. 2005. How many principal components? Stopping 340 rules for determining the number of non-trivial axes revisited. Computational Statistics and Data 341 Analysis 49:974-997. DOI: 10.1016/j.csda.2004.06.015

342 R Core Team. 2021. R: A language and environment for statistical computing. R Foundation for 343 Statistical Computing, Vienna, Austria. Available at https://www.R-project.org/ 
344 Raîche G, Magis D. 2020. An R package for parallel analysis and non-graphical solutions to the

345 Cattell scree test. R package version 2.4.1. Available at https://CRAN.R-

346 project.org/package $=$ nFactors

347 Ringnér M. 2008. What is principal component analysis? Nature Biotechnology 26:303-304.

348 DOI: $10.1038 / \mathrm{nbt} 0308-303$

349 Stauffer DF, Garton EO, Steinhorst RK. 1985. A comparison of principal components from real 350 and random data. Ecology 66:1693-1698. DOI: 10.2307/2937364

351 ter Braak CJF. 1988. CANOCO: a Fortran program for canonical community ordination by

352 [partial][detrended][canonical] correspondence analysis, principal component analysis and

353 redundancy analysis (version 2.1). Agricultural Mathematics Group, Wageningen, Netherlands.

354 Vieira VMNCS. 2012. Permutation tests to estimate significances on Principal Components

355 Analysis. Computational Ecology and Software 2:103-123.

356 Vitale R, Westerhuis JA, Næs T, Smilde AK, de Noord OE, Ferrer A. 2017. Selecting the

357 number of factors in principal component analysis by permutation testing-Numerical and

358 practical aspects. Journal of Chemometrics e2937. DOI: 10.1002/cem.2937

359 Wong MKL, Carmona CP. 2021. Including intraspecific trait variability to avoid distortion of

360 functional diversity and ecological inference: Lessons from natural assemblages. Methods in

361 Ecology and Evolution 12:946-957. DOI: 10.1111/2041-210X.13568. 


\section{Figure Legends}

363

364 Figure 1. Null distributions and empirical $\psi$ and $\varphi$ statistics derived from PCAtest analysis of a 365 single replicate of simulated data consisting of five uncorrelated variables and 100 observations. 366

367 Figure 2. Null distributions and empirical statistics derived from PCAtest analysis of a single 368 replicate of simulated data consisting of five correlated variables $(r=0.25)$ and 100 observations.

369 Lower plots show mean observed values (red dots), 95\%-confidence interval (CI) based on 1,000

370 bootstrap replicates (red bars), mean values and 95\%-CI based on 1,000 random permutations

371 (gray dots and bars, respectively).

372

373 Figure 3. Null distributions and empirical statistics derived from PCAtest analysis of a single 374 replicate of simulated data consisting of five correlated variables $(r=0.50)$ and 100 observations.

375 Lower plots show mean observed values (red dots), 95\%-confidence interval (CI) based on 1,000

376 bootstrap replicates (red bars), mean values and 95\%-CI based on 1,000 random permutations 377 (gray dots and bars, respectively).

378

379 Figure 4. Null distributions and empirical statistics derived from PCAtest analysis of seven 380 morphological variables measured in 29 ant species (data from Wang and Carmona, 2021).

381 Lower plots show mean observed values (red dots), 95\%-confidence interval (CI) based on 1,000

382 bootstrap replicates (red bars), mean values and 95\%-CI based on 1,000 random permutations 383 (gray dots and bars, respectively). 
385 Figure 5. Null distributions and empirical statistics derived from R-mode PCAtest analysis of 386 8,534 genes screened in 105 samples (data from Ringnér, 2008). Lower plots show mean 387 observed values (red dots), 95\%-confidence interval (CI) based on 1,000 bootstrap replicates (red 388 bars), mean values and 95\%-CI based on 1,000 random permutations (gray dots and bars, 389 respectively).

390

391 Figure 6. Null distributions and empirical statistics derived from PCAtest analysis of 8,534 genes 392 (observations) screened in 105 samples (variables). The original data set of Ringnér (2008) was 393 transposed to perform a Q-mode PCA analysis. Lower plots show mean observed values (red 394 dots), 95\%-confidence interval (CI) based on 1,000 bootstrap replicates (red bars), mean values 395 and 95\%-CI based on 1,000 random permutations (gray dots and bars, respectively). 
Figure 1

Null distributions and empirical statistics derived from PCAtest analysis of simulated data consisting of five uncorrelated variables and 100 observations. 

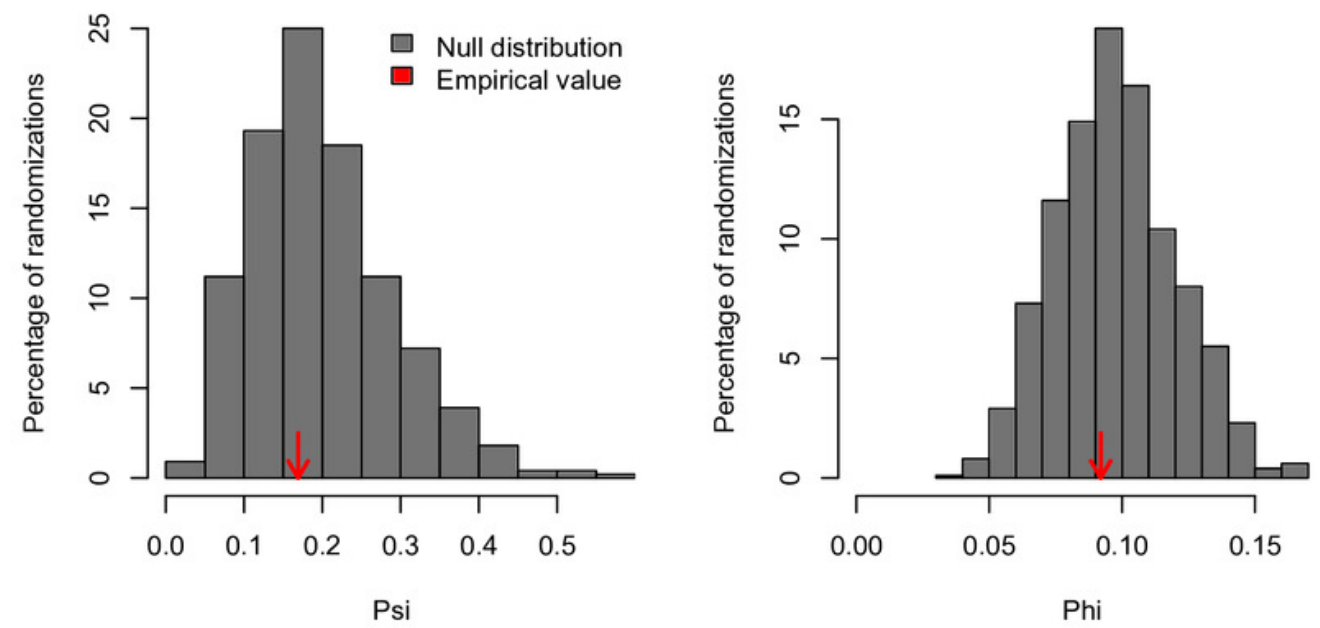
Figure 2

Null distributions and empirical statistics derived from PCAtest analysis of simulated data consisting of five correlated variables $(r=0.25)$ and 100 observations.

Lower plots show mean observed values (red dots), 95\%-confidence interval $(\mathrm{Cl})$ based on 1,000 bootstrap replicates (red bars), mean values and $95 \%-\mathrm{Cl}$ based on 1,000 random permutations (gray dots and bars, respectively). 


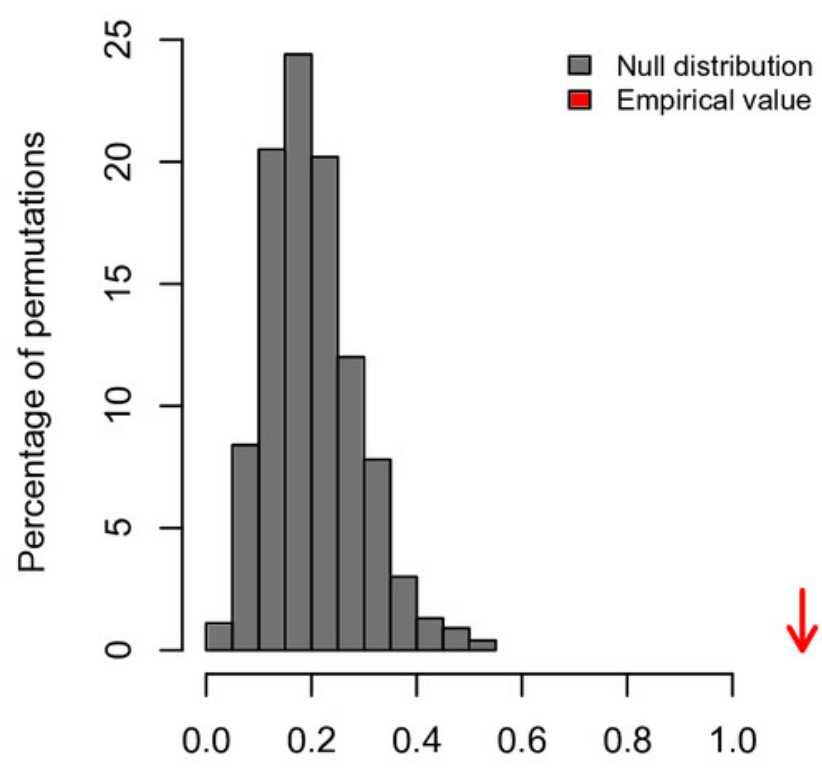

Psi

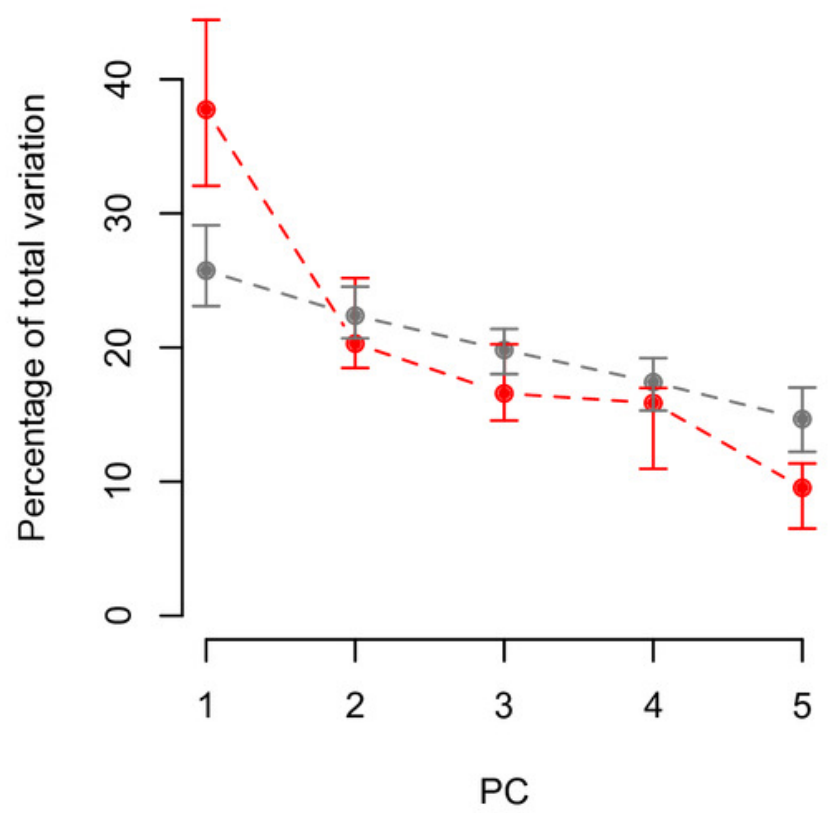

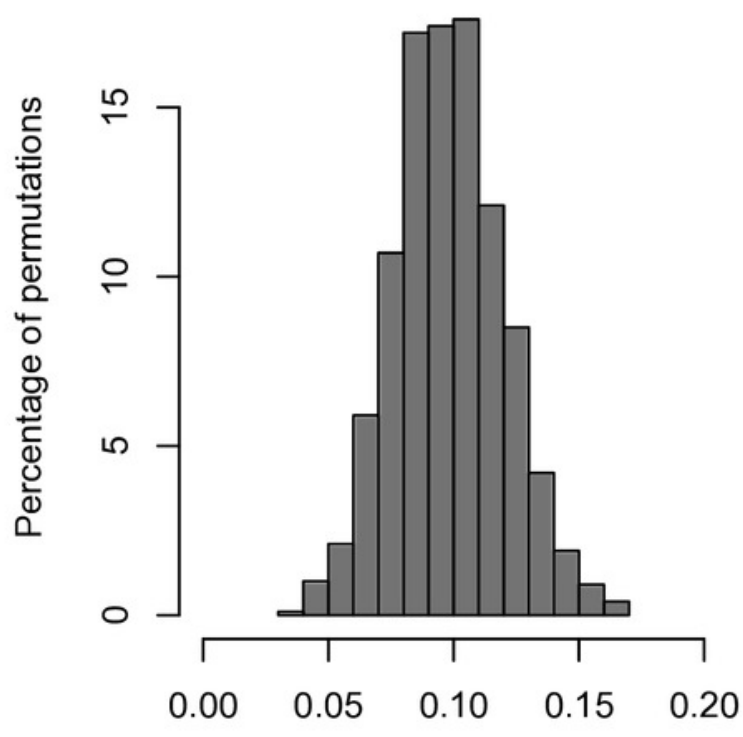

Phi

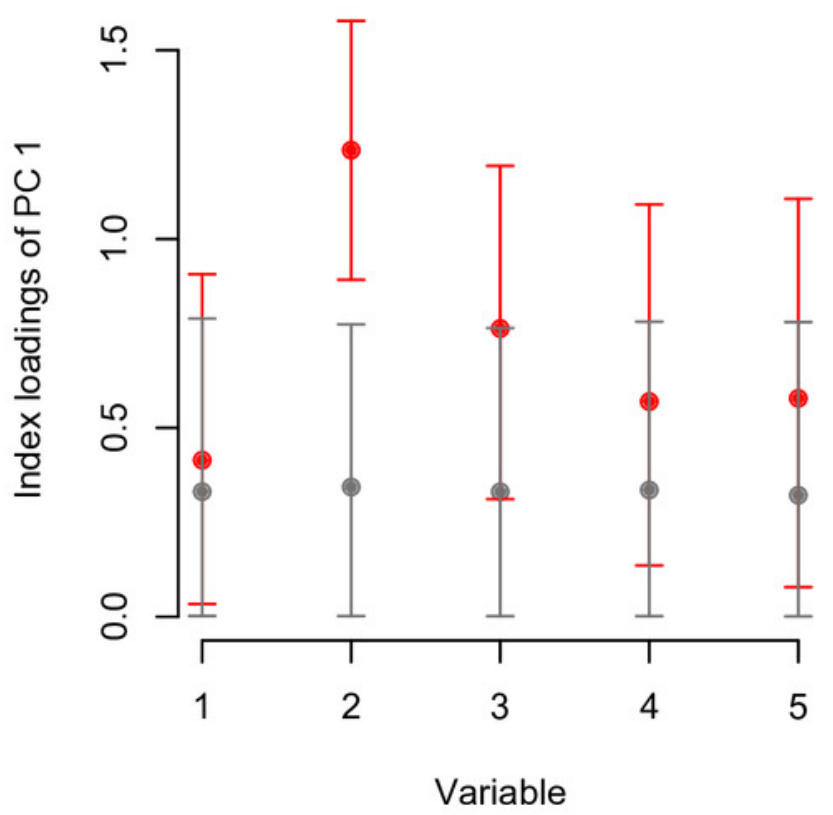




\section{Figure 3}

Null distributions and empirical statistics derived from PCAtest analysis of simulated data consisting of five correlated variables $(r=0.50)$ and 100 observations.

Lower plots show mean observed values (red dots), 95\%-confidence interval $(\mathrm{Cl})$ based on 1,000 bootstrap replicates (red bars), mean values and $95 \%-\mathrm{Cl}$ based on 1,000 random permutations (gray dots and bars, respectively). 

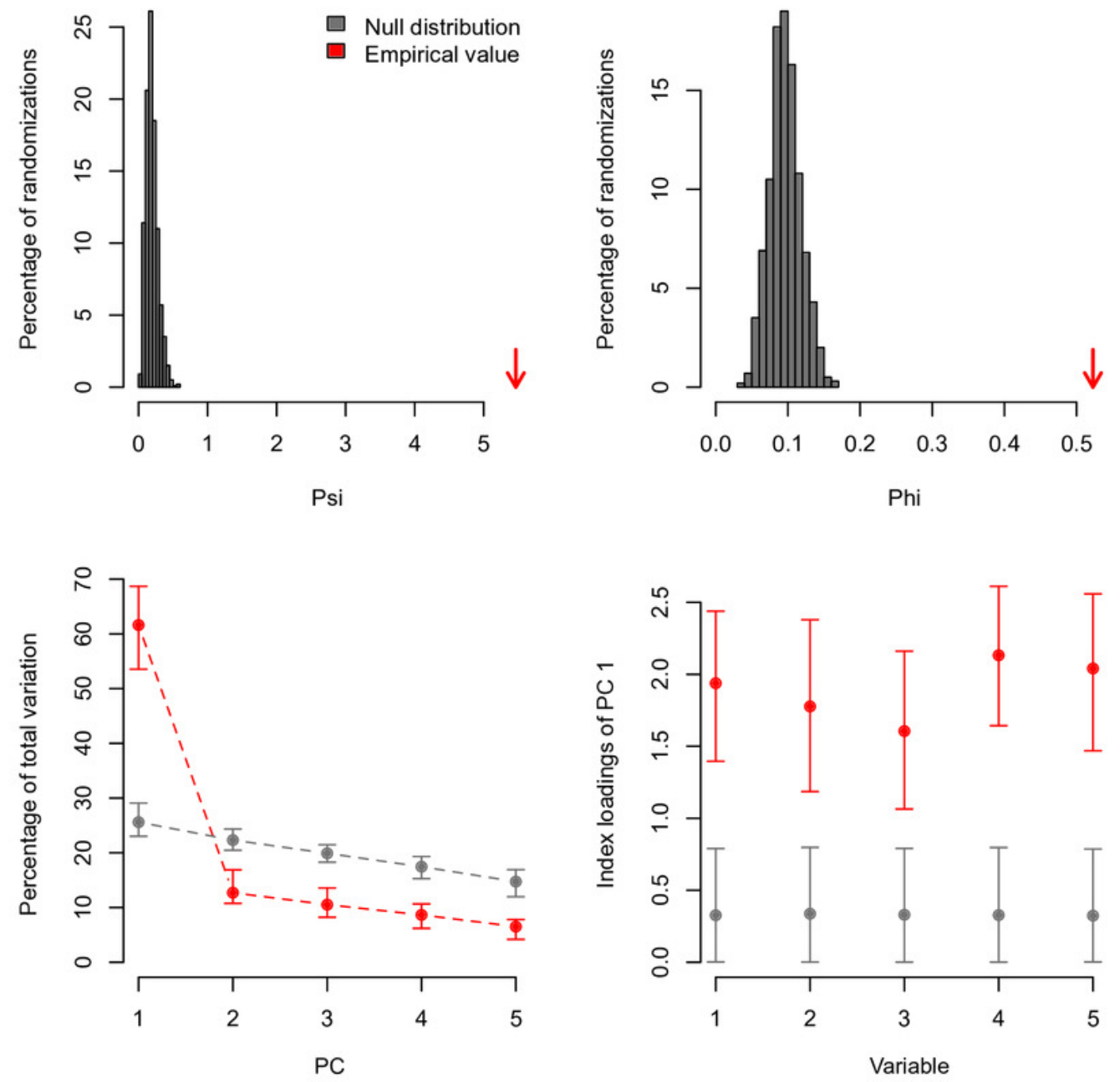
Figure 4

Null distributions and empirical statistics derived from PCAtest analysis of seven morphological variables measured in 29 ant species (data from Wang and Carmona, 2021).

Lower plots show mean observed values (red dots), 95\%-confidence interval $(\mathrm{Cl})$ based on 1,000 bootstrap replicates (red bars), mean values and $95 \%-\mathrm{Cl}$ based on 1,000 random permutations (gray dots and bars, respectively). 
(A)

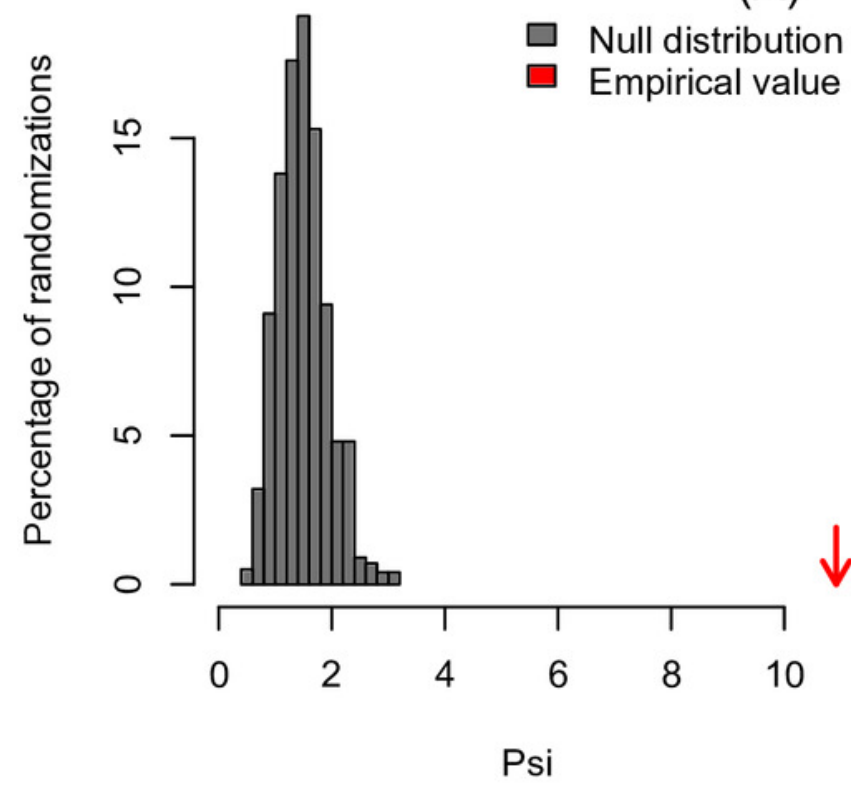

(C)

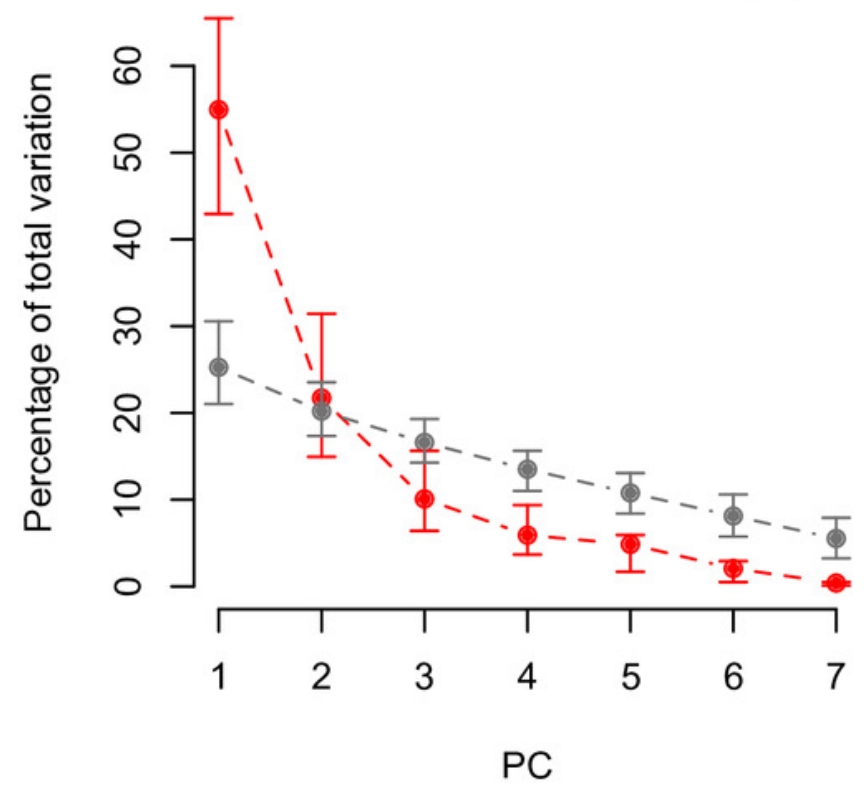

(B)

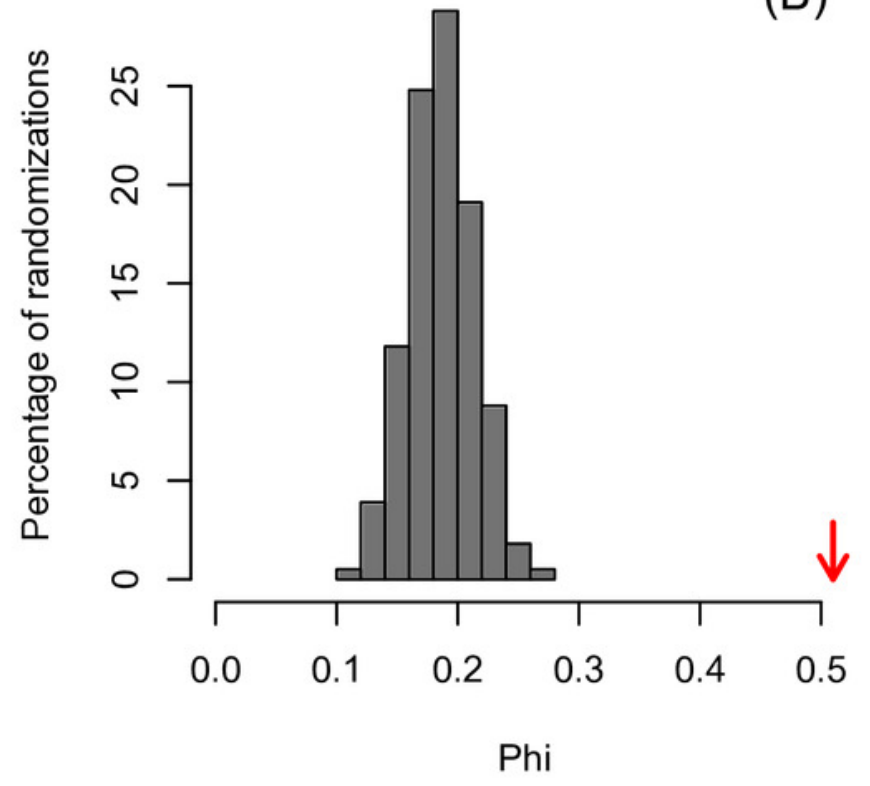

(D)

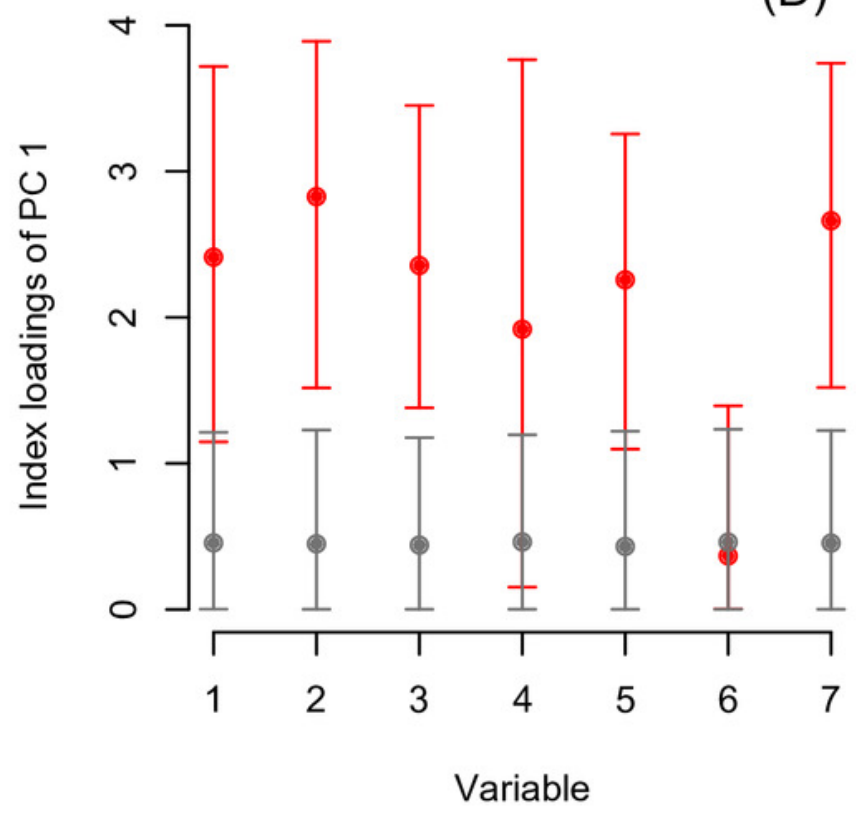




\section{Figure 5}

Results of R-mode PCAtest analysis of Ringnér (2008)'s microarray data.

Null distributions and empirical statistics derived from PCAtest analysis of 8,534 genes

screened in 105 samples (data from Ringnér, 2008). Lower plots show mean observed values (red dots), 95\%-confidence interval ( $\mathrm{Cl}$ ) based on 1,000 bootstrap replicates (red bars), mean values and $95 \%-\mathrm{Cl}$ based on 1,000 random permutations (gray dots and bars, respectively). 
(A)

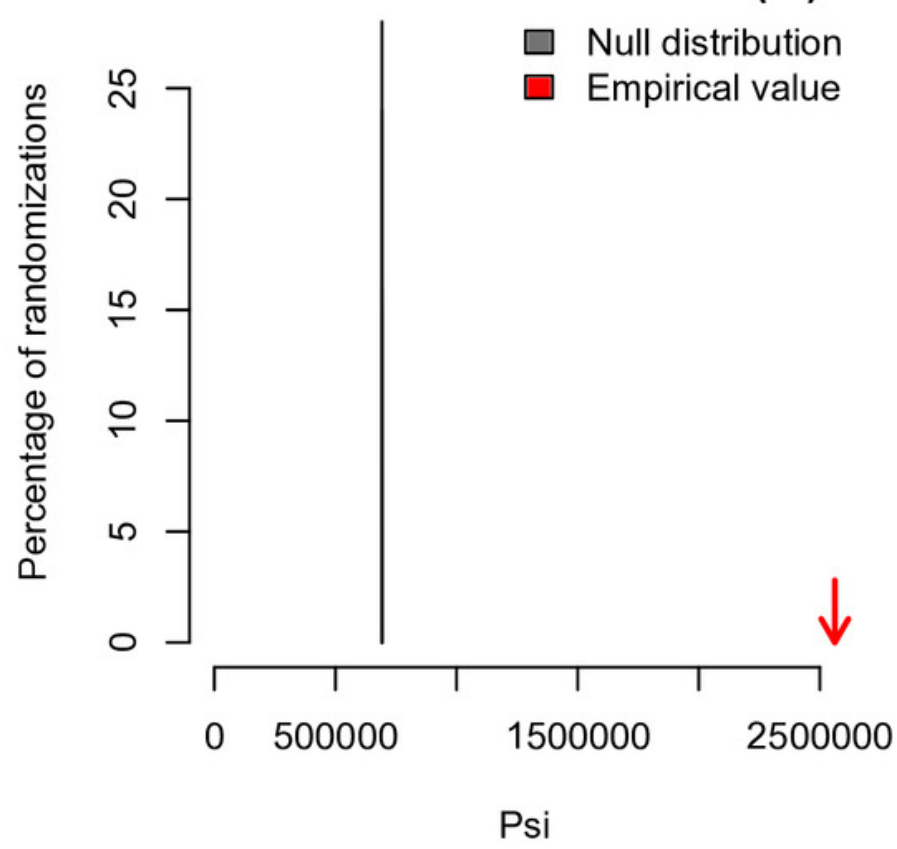

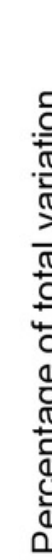

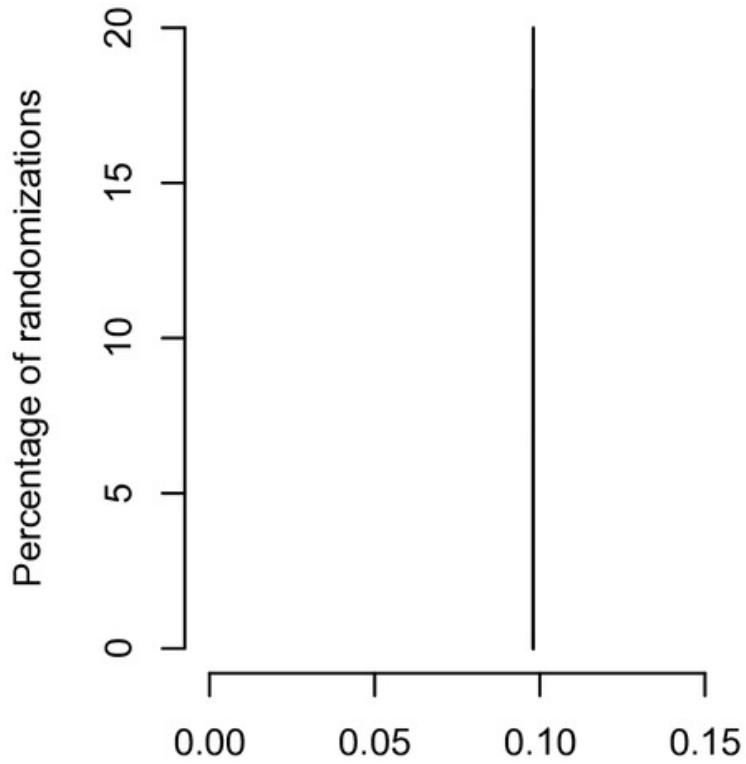

Phi
(B)

(C)

PC 


\section{Figure 6}

Results of Q-mode PCAtest analysis of Ringnér (2008)'s microarray data.

Null distributions and empirical statistics derived from PCAtest analysis of 8,534 genes (observations) screened in 105 samples (variables). The original data set of Ringnér (2008) was transposed to perform a Q-mode PCA analysis. Lower plots show mean observed values (red dots), 95\%-confidence interval $(\mathrm{Cl})$ based on 1,000 bootstrap replicates (red bars), mean values and $95 \%-\mathrm{Cl}$ based on 1,000 random permutations (gray dots and bars, respectively).
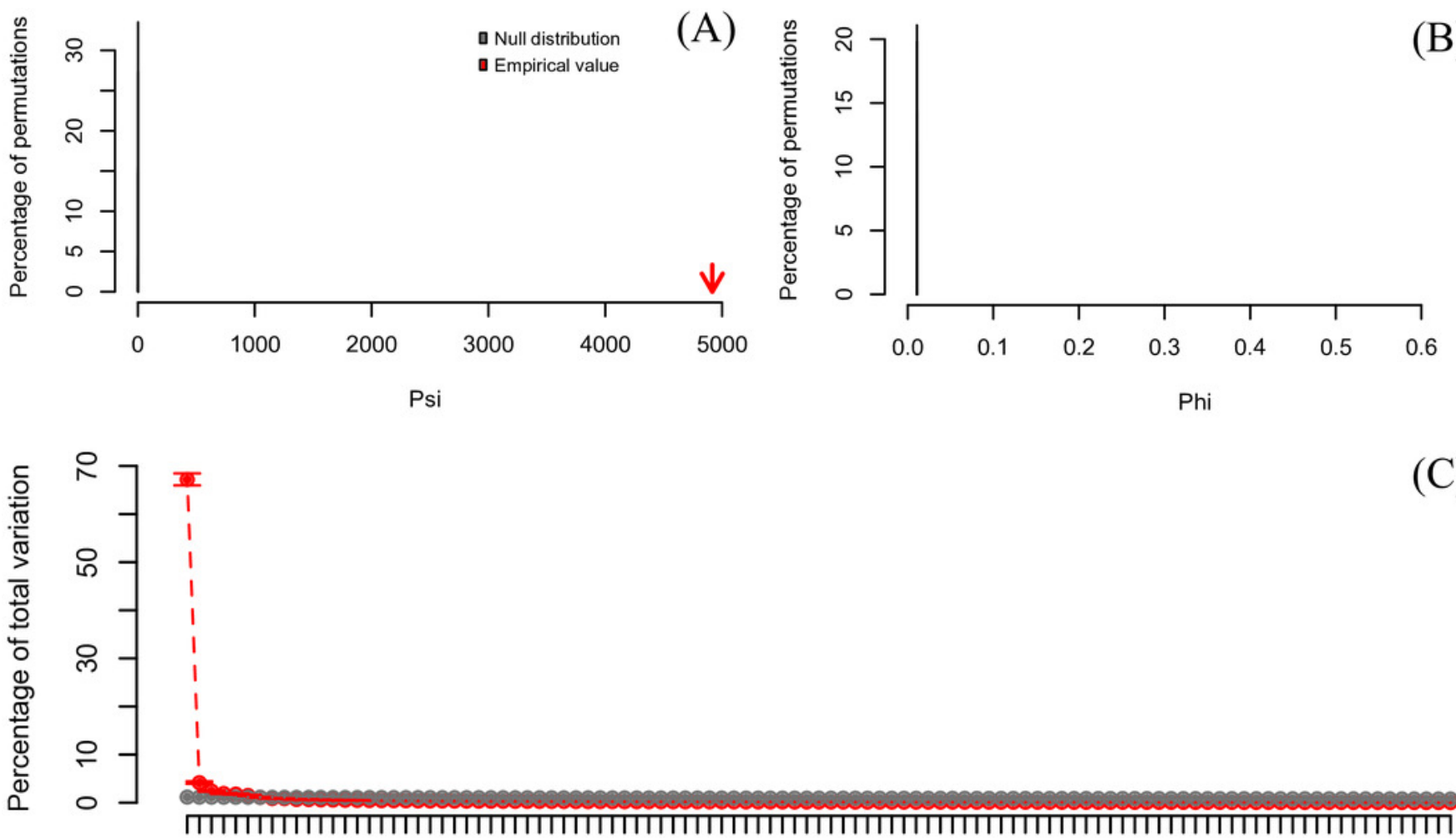

$\begin{array}{llllllllllllllllllllll}1 & 5 & 9 & 13 & 18 & 23 & 28 & 33 & 38 & 43 & 48 & 53 & 58 & 63 & 68 & 73 & 78 & 83 & 88 & 93 & 98 & 104\end{array}$

PC 


\section{Table $\mathbf{1}$ (on next page)}

Review of articles published in Peerj journals that used PCA.

Results of the review of 155 articles published in Peer) journals during 2021 (until Nov $30^{\text {th }}$ ) that used PCA. The reference list for this review is available in the Table S1. Abbreviations: Cum. var. = cumulative variance, eigen. = eigenvalue. 
1 Table 1: Results of the review of 155 articles published in PeerJ journals during 2021 (until Nov $30^{\text {th }}$ ) that 2 used PCA. The reference list for this review is available in the Table S1. Abbreviations: Cum. var. = cumulative variance, eigen. $=$ eigenvalue.

\begin{tabular}{lccccc}
\hline \multicolumn{1}{c}{$\begin{array}{c}\text { Journal } \\
\text { Section }\end{array}$} & \multicolumn{3}{c}{ Criteria for PC selection } & Total \\
\cline { 2 - 5 } & None & Cum.var. & Eigen. $>1$ & Scree plot & \\
\hline PeerJ Life \& Environment & 117 & 12 & 11 & 1 & 141 \\
\cline { 2 - 5 } Aquatic Biology & 11 & 0 & 0 & 0 & 11 \\
Biochemistry, Biophysics \& Molecular Biology & 10 & 1 & 0 & 0 & 11 \\
Biodiversity \& Conservation & 4 & 1 & 2 & 0 & 7 \\
Bioinformatics \& Genomics & 24 & 1 & 0 & 1 & 26 \\
Brain, Cognition \& Mental Health & 1 & 1 & 1 & 0 & 3 \\
Ecology & 7 & 2 & 0 & 0 & 9 \\
Environmental Science & 3 & 0 & 1 & 0 & 4 \\
Microbiology & 12 & 2 & 1 & 0 & 15 \\
Paleontology \& Evolutionary Science & 3 & 2 & 0 & 0 & 5 \\
Plant Biology & 28 & 0 & 4 & 0 & 32 \\
Zoological Science & 11 & 2 & 1 & 0 & 14 \\
None assigned & 3 & 0 & 1 & 0 & 4 \\
PeerJ Computer Science & 12 & 2 & 0 & 0 & 14 \\
\hline Total & 129 & 14 & 11 & 1 & 155 \\
\hline
\end{tabular}

5 